\title{
Erratum: Optimal Continuous Variable Quantum Teleportation with Limited Resources [Phys. Rev. Lett. 119, 120503 (2017)]
}

\author{
Pietro Liuzzo-Scorpo, Andrea Mari, Vittorio Giovannetti, and Gerardo Adesso
}

(Received 28 November 2017; published 12 January 2018)

DOI: 10.1103/PhysRevLett.120.029904

The Letter was published with an error in Eq. (9), leading to unphysical states when $\tau>1$. This resulted in some incorrect statements regarding the finite-energy simulation of the quantum limited amplifier channel. No other results in the Letter, including those in the section on optimal teleportation fidelity, are affected by this error. In the following, we provide the correct version of Eq. (9), leading to physical states that can be used to simulate both attenuators $(\tau<1)$ and amplifiers $(\tau>1)$, as well as a revision of the affected statements.

In Eq. (9), the inequality

$$
b \geq \frac{\tau-e^{-2 r} \tanh r}{\tau-\tanh r}
$$

should be replaced by

$$
b \geq \frac{e^{2 r} \tau+e^{-2 r}-|\tau-1|}{\tau+1-e^{2 r}|\tau-1|} .
$$

In the abstract of the Letter, the sentence "We first characterize the class of single-mode phase-insensitive Gaussian channels that can be simulated via a Braunstein-Kimble protocol with nonunit gain and minimum shared entanglement, showing that infinite energy is not necessary apart from the special case of the quantum limited attenuator" should be replaced by "We first characterize the class of single-mode phase-insensitive Gaussian channels that can be simulated via a Braunstein-Kimble protocol with nonunit gain and minimum shared entanglement, showing that infinite energy is not necessary apart from the special cases of the quantum limited attenuator and amplifier".

In the introduction, right column of page 1, the sentence "We show that almost all of them (but the quantum limited attenuator) can be implemented by teleportation with more realistic resource states, having the same entanglement as the Choi state but finite mean energy" should be replaced by "We show that almost all of them (but the quantum limited attenuator and amplifier) can be implemented by teleportation with more realistic resource states, having the same entanglement as the Choi state but finite mean energy".

In the section on implementable phase-insensitive channels, right column of page 3, the text "with the exclusion of only one point, i.e., the quantum limited attenuator" should be replaced by "with the exclusion of only two points, i.e., the quantum limited attenuator and amplifier".

In the same section, the end of page 3 through to the beginning of page 4, the sentences "Notice that the lower bound on the coefficient $b$ in Eq. (9) (and, hence, $a$ ) diverges only at the extreme point ( $\tau=\tanh r, y=1-\tanh r$ ) corresponding to the quantum limited attenuator. For larger values of $\tau$, including the opposite extreme represented by the quantum limited amplifier, all channels along the boundary saturating Eq. (8) can be simulated using the states (9) with finite mean energy $\bar{n}_{A B}$ and minimum entanglement $E_{\mathcal{N}}=2 r$ (see Fig. 1)" should be replaced by "Notice that the lower bound on the coefficient $b$ in Eq. (9) (and, hence, $a$ ) diverges only at the two extreme points ( $\tau=\tanh r, y=1-\tanh r$ ), corresponding to the quantum limited attenuator, and ( $\tau=\operatorname{coth} r, y=\operatorname{coth} r-1)$, corresponding to the quantum limited amplifier. For all the intermediate values of $\tau$ in the accessible range $\tanh (r)<\tau<\operatorname{coth}(r)$, all channels along the boundary saturating Eq. (8) can be simulated using the states (9) with finite mean energy $\bar{n}_{A B}$ and minimum entanglement $E_{\mathcal{N}}=2 r$ (see Fig. 1)".

In the same section, left column of page 4, the sentence "Quite surprisingly, in this case the energy (and the entanglement) stays finite even for the quantum limited attenuator and diverges only for the identity map $(\tau=1, y=0)$ " should be replaced by "Quite surprisingly, in this case the energy (and the entanglement) stays finite even for the quantum limited attenuator and amplifier and diverges only for the identity map $(\tau=1, y=0)$ ".

In the conclusions, left column of page 5, the text "Excluding the pathological case of the quantum limited attenuator" should be replaced by "Excluding the pathological cases of the quantum limited attenuator and amplifier".

We are grateful to Riccardo Laurenza and Stefano Pirandola for noticing the error in Eq. (9) of the published Letter. 